\title{
Dysphagia Post-Extubation
}

\author{
Jennifer Hanners, MS, CCC-SLP
}

\section{CASE}

A 68-year-old man required mechanical ventilation for five days during an acute flare in his COPD. His voice is slightly hoarse post extubation. Does he need a speech therapy evaluation?

\section{Discussion}

Acute care speech pathologists are often consulted to evaluate swallowing following extubation. Swallowing is an intricate process "requiring the precise timing and coordination of more than 25 muscles" and engaging 5 cranial nerves. ${ }^{1}$ Intubation may negatively impact pharyngeal and laryngeal proprioception; it may also affect the strength of hyolaryngeal elevation needed for optimal upper esophageal sphincter (UES) opening and epiglottal inversion during swallow and pharyngeal contraction needed for propulsion of the bolus to the upper esophagus. Factors such as length of endotracheal intubation and age may influence incidence of post-extubation swallow dysfunction [PSD]. A study conducted in 2011 commented, "Aspiration is the leading cause of nosocomial infection in critically ill populations." This same study detected a dysphagia incidence of $41 \%$ in patients intubated 48 hours or more and cited "oropharyngeal muscle inactivity, glottic injury, mucosal inflammation leading to loss of architecture, and vocal cord ulceration" as potential contributors to PSD. ${ }^{1}$ Based on these findings, the authors advised early dysphagia intervention by speech pathology. This particular study did not identify co-morbidities as a predictor of PSD.

\section{Corresponding author: Jennifer Hanners, MS,} CCC-SLP

Contact Information: Jenneifer.hanners@umchealthsystem.com

DOI: $10.12746 /$ swrccc2013.0103.028
Another study concluded that "intubation of more than 1 day was an independent risk factor" for development of dysphagia and concurrent empirical evidence indicates "moderate or severe dysphagia is associated with increased risk of reintubation, development of pneumonia, longer hospital stay, reduced dietary intake...." ${ }^{2}$ Macht and coworkers reported a dysphagia incidence of $84 \%$ in patients retrospectively analyzed from 2008-2010 who received mechanical ventilation via endotracheal intubation for any amount of time. ${ }^{3}$

Speech pathologists, in many cases, can detect swallow dysfunction through a bedside/clinical swallow examination. This type of evaluation does not involve use of equipment. The speech pathologist chooses various food and liquid consistencies to determine a patient's aptitude for a safe swallow. Often, a graham cracker, ice cream or pudding, honey-thick liquid, nectar-thick liquid, and thin liquid are utilized to analyze function. If the patient is poorly responsive or still in some distress, a limited screening with ice chips, water, and thickened water is more appropriate. During this examination, the speech pathologist analyzes many different indicators of swallow dysfunction. Although many consider coughing the "gold standard" for detecting deficient swallow capabilities, a cough may indicate better laryngeal sensory perception of a food/liquid stimulus entering the laryngeal vestibule. Conversely, no coughing may indicate a patient's inability to detect aspirated material due to deficient sensation.

One initial point of analysis is the time it takes to manipulate the bolus from the anterior to posterior oral cavity. Oral motor range of motion and strength of the oral, lingual, and facial musculature is also observed to determine the patient's potential for effective chewing. Once the swallow is initiated, palpation of the thyroid cartilage and the surrounding struc- 
tures/tissue reveals subjective strength of laryngeal elevation [superior movement of the larynx to contribute to epiglottal inversion for protection of the trachea during swallowing] and laryngeal excursion [anterior movement of the hyoid to contribute to enlargement of the pharynx]. Vocal quality post swallow is a key indicator of swallow safety or possible dysfunction. If a portion of the bolus penetrates into the laryngeal vestibule, residue on the vocal folds will be evident during speaking. Other points of analysis include respiratory rate and quality of breathing post swallow. If a patient has a tracheostomy, food particles exiting the hub of the tracheostomy post swallow and evidence of food/ liquid revealed during deep suctioning are important signs of deficient swallow capability.

In some cases, the bedside/clinical swallow examination is not enough to determine presence of swallow dysfunction. Silent aspiration [aspiration with no subjective indicator of swallow dysfunction] does occur in some medical diagnosis, such as COPD, and with age. 4,5 Objective testing via Modified Barium Swallow or Fiberoptic Endoscopic Evaluation of Swallowing [FEES] may be warranted if silent aspiration is suspected as a potential contributor chronic pulmonary disease, weight loss, or discomfort during PO feedings. The Modified Barium Swallow is a radiographic study, completed with fluoroscopy. This study is excellent at analyzing amount of aspiration, effectiveness of compensatory strategies used to reduce aspiration, and functional and organic differences between right and left oral-pharyngeal planes. In addition, if an esophageal abnormality is suspected, a fluoroscopic sweep of the esophagus may reveal evidence of esophageal dysfunction as a contributor to dysphagia. The FEES examination incorporates use of an endoscope to view the larynx before and immediately after the swallow, analyzing for potential presence of food or liquid in the laryngeal vestibule or on the vocal folds following the swallow. The FEES examination is helpful when the patient cannot be transported to radiology, when radiation is a concern, or when examination of the tissue quality is essential, such as a patient with fibrosis following radiation treatment for cancer.

If deficits in swallow function are detected during a bedside/clinical or objective swallow examination, several therapeutic techniques can help rehabilitate swallow function. A speech pathologist may prescribe an oral-pharyngeal exercise regime to improve the strength of swallowing. Techniques, such as swallowing repeatedly with the tongue held gently between the teeth or performing isometric and isokinetic head raises from a supine position, assist in heightening laryngeal elevation. Other techniques, such as repeated tongue protrusion followed by an effortful swallow, improve anterior movement of the posterior pharyngeal wall toward the base of tongue for stronger bolus propulsion. For recovery of the sensory component of swallowing, cold-temperature [thermal] stimulation of areas of the oral cavity rich in sensory receptors improve responsiveness to bolus presentations and optimize timing of the swallow.

Speech pathology intervention can be beneficial for many reasons beyond determining if a swallow is "safe" or not. While a patient takes food or liquid by mouth, a speech pathologist can help examine the need for: adaptive feeding equipment, various head or body positions to maximize effective PO consumption, use of compensatory strategies to reduce risk of aspiration, oral pain control or modified diet consistencies to produce greater comfort with $\mathrm{PO}$ feedings.

Our patient could not safely drink liquids. After a bedside/clinical examination detected several subtle signs of dysphagia, including a mild change in vocal quality post swallow and a delayed nonproductive cough, a Modified Barium Swallow revealed consistent aspiration of thin liquid. His diet was modified to use thickened liquids. After three days of dysphagia therapy, his swallowing was re-evaluated by a speech pathologist, and he was placed on a regular diet.

\section{KEY POINTS}

1. Dysphagia occurs frequently after mechanical ventilation using endotracheal tubes.

2. Dysphagia increases the risk of aspiration and reduces calorie intake. 
3. Speech pathologists can quickly evaluate patients at the bedside and facilitate rehabilitation.

KEY WORDS- dysphagia, speech pathologist, airway extubation

Author Affiliation: Jennifer Hanners is a Speech therapist at University Medical Center, Lubbock TX.

Received: $11 / 13 / 2012$

Accepted: 6/10/2013

Reviewers: Kenneth Nugent MD, Zachary Mulkey MD

Published electronically: 7/15/2013

Conflict of Interest Disclosures: None

\section{REFERENCES}

1. Bordon A, Bokhari R, Sperry J, Testa D, Feinstein A, Ghaemmaghami V. (2011). Swallowing dysfunction after prolonged intubation: analysis of risk factors in trauma patients. The American Journal of Surgery,202: 679-683.

2. Takeuchi A, Taniguchi T, Ishii Y, Izumi M, Furuya K, Kato K, Maekawa K, Asai Y. (2011). Association between length of intubation and development of dysphagia in critically ill patients. Critical Care Medicine, 39 (supplement 12): 159.

3. Macht M, Wimbish T, Clark BJ, Benson AB, Burnham EL, Williams A, Moss M. (2011). Postextubation dysphagia is persistent and associated with poor outcomes in survivors of critical illness. Critical Care, 15: R231.

4. Smith CH, Logemann JA, Colangelo LA, Rademaker AW, Pauloski BR. (1999). Incidence and patient characteristics associated with silent aspiration in the acute care setting. Dyspahgia, 14(1):1-7.

5. Kikuchi R, Watabe N, Konno T, Mishina N, Sekizawa K, Sasaki H. (1994). High incidence of silent aspiration in elderly patients with community-acquired pneumonia. American Journal of Respiratory Critical Care Med, 150: 251-253. 